\title{
Stenting of the arterial duct: a new approach to palliation for pulmonary atresia
}

John L Gibbs, Martin T Rothman, Michael R Rees, Jonathan M Parsons, Mike E Blackburn, Carlos E Ruiz

\begin{abstract}
Objective-To assess the possibility of maintaining ductal patency in neonates with complex pulmonary atresia by percutaneous implantation of balloon expandable stents.

Patients-Two duct-dependent neonates with long segment pulmonary atresia, right sided aortic arch, and left sided arterial duct.

Results-Stents with final diameter of 3.5 or $4 \mathrm{~mm}$ and initial length of 7 or $15 \mathrm{~mm}$ were successfully positioned in the arterial duct. Two stents were required in one child and four in the other in order to stent the entire length of the duct. After the procedures the ducts remained widely patent and arterial oxygen saturations remained above $80 \%$. Complications of the procedures included perforation of a peripheral pulmonary artery and cardiac perforation, both caused by guide wire manipulation. Both babies died suddenly, one at five weeks, and the other at nine days after successful stenting of the duct. Both ducts were patent at necropsy; the exact cause of one death was not clearly defined, but the second seemed to be caused by pneumococcal septicaemia.
\end{abstract}

Conclusions-Stenting of the arterial duct is technically feasible. It provides adequate palliation for neonates with pulmonary atresia at least in the short term and it seems to result in balanced, central perfusion of both pulmonary arteries. This preliminary report suggests that this previously untried technique may prove to be a promising and attractive alternative to neonatal aortopulmonary shunt operation.

Killingbeck Hospital,
Leeds
J L Gibbs
M R Rees
J M Parsons
M E Blackburn
The Royal London
Hospital, Whitechapel,
London
M T Rothman
University of Loma
Linda, California,
USA
C E Ruiz
Correspondence to
Dr J Gibbs, Department
of Paediatric Cardiology,
Killingbeck Hospital, York
Road, Leeds LS14 6UQ.

Conventional palliative treatment of duct dependent long segment pulmonary atresia consists of treatment with prostaglandin $\mathrm{E}$ in the short term and of surgical fashioning of an aortopulmonary shunt in the medium term. A logical alternative to surgical aortopulmonary anastomosis would be to prevent the arterial duct from closing. Expandable metal stents have proved effective in the treatment of intravascular stenoses at various sites including the pulmonary arteries and the inferior caval vein ${ }^{1}$ and, more recently, in coronary artery stenosis or intimal dissection after balloon angioplasty. ${ }^{2}$ Stenting of the arterial duct in duct dependent congenital heart disease has not, however, been attempted. We report the successful maintenance of ductal patency by implantation of expandable stainless steel stents. The technical procedure (performed in each case under general anaesthesia), its potential complications, and its very considerable future promise in the palliation of duct dependent congenital heart disease are discussed.

\section{Patients and methods}

CASE 1

A $2.6 \mathrm{~kg}$ boy presented with cyanosis and a soft continuous murmur when he was four hours old. He had an extensive cleft lip and palate and a bifid right thumb. Echocardiography and later cardiac catheterisation and angiography showed severe hypoplasia of the right ventricle and tricuspid valve with an intact ventricular septum, right ventricular sinusoids, long segment pulmonary atresia, small but confluent pulmonary arteries, a right sided aortic arch, and a left sided arterial duct arising at the origin of the left subclavian artery. He was maintained on an infusion of prostaglandin $\mathrm{E}$ and at the age of five days underwent a Waterston aortopulmonary anastomosis. The right pulmonary artery was found to be approximately $2.5 \mathrm{~mm}$ in diameter behind the ascending aorta. Postoperatively the child remained duct dependent. Angiography showed that the shunt was completely occluded. In view of the failed attempt at surgical palliation and with informed consent from the child's parents, we believed the alternative approach of stenting the arterial duct was justified.

Initially we chose a titanium wire stent (William Cook, USA) that was premounted on a balloon catheter with $4 \mathrm{~mm}$ inflated diameter and a $5 \mathrm{~F}$ shaft. The $6 \mathrm{~F}$ sheath necessary for delivery of the device, however, proved too large to pass retrogradely up the femoral artery or anterogradely to the duct via the inferior caval vein, the atrial septum, the left ventricle and the ascending aorta. Clearly, it was essential to use a device that could be delivered through a smaller sheath and we then chose a Palmaz-Schatz stainless steel stent (Ethicon Ltd, Johnson \& Johnson) designed for implantation in adult coronary arteries (fig 1). ${ }^{12}$ This stent is $15 \mathrm{~mm}$ long, consisting of two $7 \mathrm{~mm}$ lengths joined by a single $1 \mathrm{~mm}$ bridge, the two halves being 
Figure 1 A 7 mm long Palmaz-Schatz coronary stent $(A)$ unexpanded, mounted upon a $3 \cdot 7 F$ 4 mm coronary angioplasty balloon catheter and (B) after inflation to a diameter of $4 \mathrm{~mm}$.
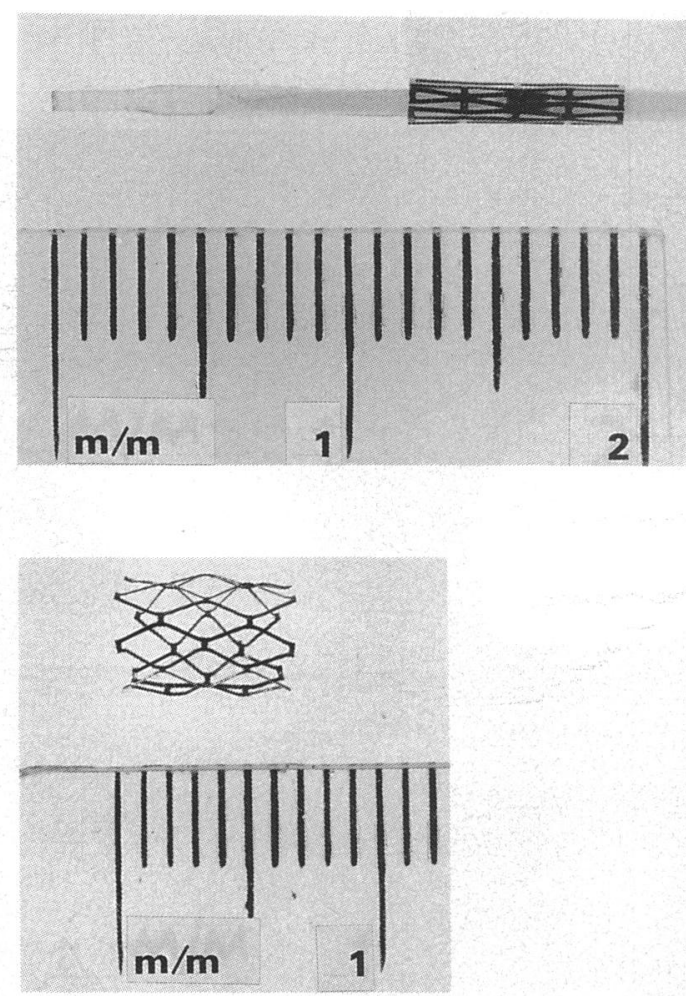

easily separated into equal $7 \mathrm{~mm}$ lengths by very careful cutting of the bridge (although this is not recommended by the manufacturer). After inflation to $4 \mathrm{~mm}$ diameter the $7 \mathrm{~mm}$ length shortens to approximately $6 \mathrm{~mm}$ and the $15 \mathrm{~mm}$ long complete stent shortens to approximately $13 \mathrm{~mm}$. The stent is operator mounted on a balloon catheter of the operator's choice, in this case an ACS coronary angioplasty balloon catheter with an inflated diameter of $4 \mathrm{~mm}$ and shaft of 3.7 F.

At right axillary artery cutdown a $5 \mathrm{~F}$ Van Andel sheath (William Cook) was passed to the aortic arch. A 4F multipurpose catheter was passed through the sheath, bleeding being prevented by an ACS Tuohy-Borst type rotating haemostatic valve. A superfloppy 0.016 inch guide wire was manipulated down the duct and into the peripheral left lower lobe pulmonary artery. The catheter was then advanced over the guide wire into the distal pulmonary artery, the guide wire was exchanged for a stiffer wire (intermediate 0.016 inch, USCI Bard) and the multipurpose catheter was replaced by the balloon catheter with the stent mounted upon it. The balloon catheter was positioned (dye injected through the sheath and the central balloon marker were used to guide accurate placement) in the duct and the balloon was inflated to $4 \mathrm{~mm}$ at a pressure of $8 \mathrm{~atm}$ for 20 seconds. There was transient hypotension and a fall in oxygen saturation during balloon inflation, with prompt recovery after deflation. Repeat angiography showed the duct to be widely patent and pulse oximetry showed an increase in peripheral oxygen saturation from $75 \%$ to $90 \%$. The stent was not visible on radiographic screening, but this was not considered remarkable because the stent is rarely visible in adult coronary arteries. Treatment with prostaglandin E was stopped and the arteriotomy repaired.

After three hours the oxygen saturation fell to $40 \%$. This did not improve after thrombolytic treatment but rose again to $85 \%$ when intravenous prostaglandin was restarted. Doppler colour flow mapping showed evidence of distal ductal stenosis which suggested that the implanted stent was too short. An attempt at changing to oral prostaglandin was unsuccessful.

Ten days later a second right axillary arteriotomy was performed and a $7 \mathrm{~mm}$ Johnson $\&$ Johnson stent was positioned in a similar manner in the distal duct uneventfully using a similar $4 \mathrm{~mm}$ balloon catheter (fig 2). Angiography confirmed a very satisfactory stent position in the distal part of the duct, with the stent being just visible on screening and plainly visible on the plain chest radiograph on this occasion (fig 2). The duct was widely patent and the arterial oxygen saturation had again risen to $90 \%$. The child was returned to the intensive care unit, where 15 minutes later the oxygen saturation again fell to $40 \%$, there was a fresh haemorrhage from the endotracheal tube and radiographic evidence of right upper lobe consolidation. The bleeding stopped without specific treatment but the next day oxygen saturation had not improved. Thrombolytic treatment did not cause any further haemorrhage but failed to produce any clinical improvement and prostaglandin $\mathrm{E}$ had little effect in the dose previously used successfully. Ultrasound examination proved unhelpful on this occasion. A repeat chest radiograph, however, showed the distal stent clearly, but the longer stent was still not visible in the proximal duct or in any other position in the vascular system. Retrospectively it became clear that the first stent had not, in fact, been deployed in the duct and had been pushed backwards off the balloon during the introduction of the balloon catheter, later to be unwittingly discarded inside the haemostatic valve.

The child improved with high dose intravenous prostaglandin $\mathrm{E}$. His parents encouraged us to reattempt stenting of the proximal duct, and three days later the child was returned to the catheter laboratory where, through a left axillary artery cutdown, a $15 \mathrm{~mm}$ stent was easily positioned and expanded to $4 \mathrm{~mm}$ in the proximal duct overlapping the distal stent by $1.5-2 \mathrm{~mm}$. Repeat angiography showed the entire duct to be widely patent and both stents were visible on screening and on the plain chest radiograph (fig 2). The oxygen saturations remained above $85 \%$ without prostaglandin E. The child was treated with dipyridamole, aspirin, and a heparin infusion pending stabilisation of the prothrombin time on warfarin.

His recovery was complicated by a further pulmonary haemorrhage five days later, requiring reversal of anticoagulation with fresh frozen plasma and discontinuation of warfarin. He continued to be treated with aspirin $15 \mathrm{mg} / \mathrm{kg}$ once daily and dipyridamole 


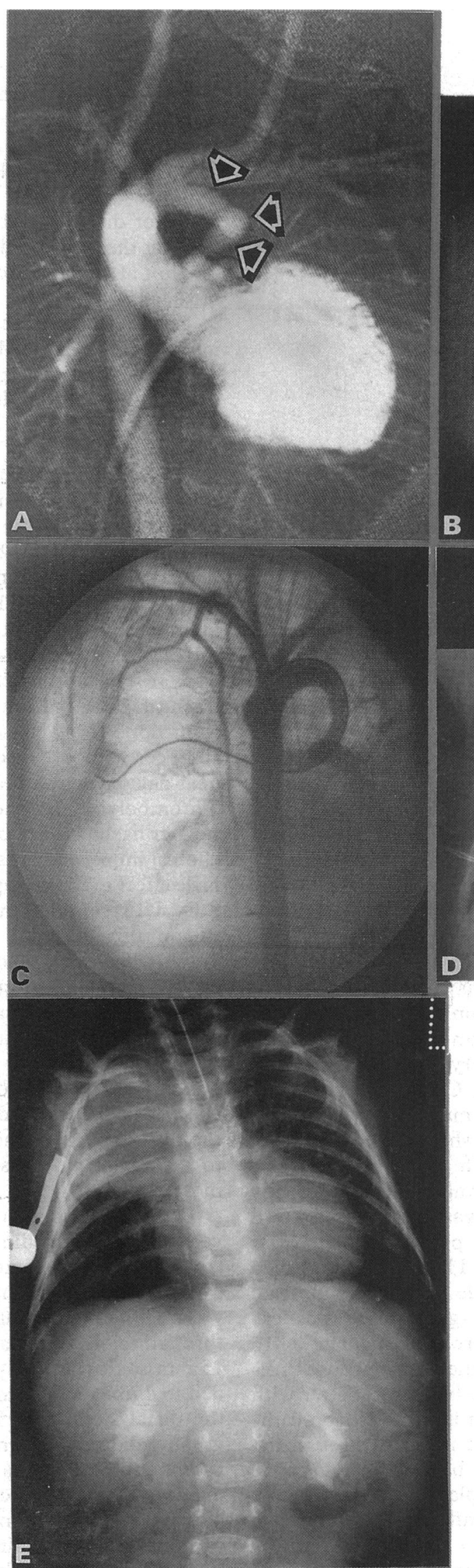

$5 \mathrm{mg} / \mathrm{kg}$ in three divided doses daily. He was extubated 11 days later, when pulse oximetry showed stable oxygen saturations between $80 \%$ and $85 \%$. Four weeks after the procedure he remained well saturated but mildly tachypnoeic, requiring treatment with diuretics. He was found dead in his cot when

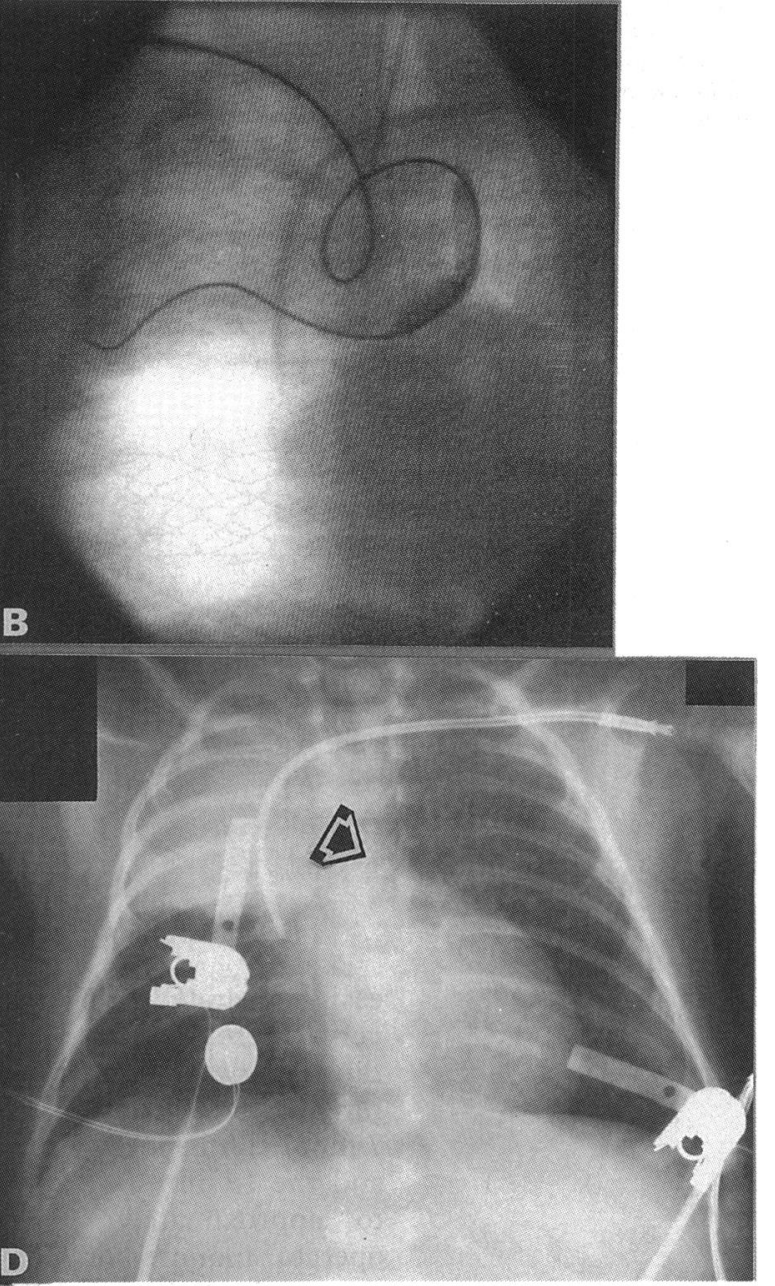

Figure 2 (A) A left ventriculogram in the anteroposterior projection showing the right sided aortic arch and the left sided arterial duct

(arrowed) that supplied the pulmonary arteries in case 1. (B) Delivery of the first $(7 \mathrm{~mm})$ stent to the distal part of the duct. The balloon is seen inflated within the duct, having been passed over a guide wire inserted via the right axillary artery and positioned in a distal pulmonary artery in the right upper lobe. (C) After deflation of the balloon, angiography showed the duct to be widely patent. (D) A plain radiograph after the delivery of the first stent showing the expanded stent to be just visible (arrowed) in the distal part of the duct. There is right upper lobe shadowing caused by pulmonary haemorrhage induced by the guide wire. E) A plain radiograph taken after completion of the procedures, showing the $7 \mathrm{~mm}$ and $15 \mathrm{~mm}$ stents expanded within the duct. The two stents overlap by $1.5-2 \mathrm{~mm}$ to produce an overall stent length of approximately $17 \mathrm{~mm}$

he was five weeks old. At necropsy the duct and both branch pulmonary arteries were widely patent and the immediate cause of death was uncertain.

A $2.6 \mathrm{~kg}$ boy presented with cyanosis when he 
was two days old. He was the second of twins (his twin brother had coarctation of the aorta). Investigation showed him to have atrial isomerism, a complete atrioventricular septal defect, and long segment pulmonary atresia with confluent but small pulmonary arteries and with a right sided aortic arch and a left sided arterial duct supplying the pulmonary arteries. His parents wished to avoid surgical treatment and gave their informed consent to an attempt at stenting the arterial duct. Angiography via a right axillary artery cutdown and a $5 \mathrm{~F}$ Van Andel sheath confirmed a right sided aortic arch with a long, curved, distally stenosed duct arising from the left side of the proximal aortic arch. The left subclavian artery arose anomalously from the descending aorta. The arterial duct was cannulated with a 4F Cobra catheter, a 0.014 inch guide wire was positioned in the right lower lobe pulmonary artery, and a $7 \mathrm{~mm}$ long Johnson \& Johnson stent was deployed in the distal duct through a $4 \mathrm{~mm}$ (3.3F) ACX coronary angioplasty balloon catheter. A brief attempt was made to insert a second stent more proximally in the duct, but the procedure was abandoned because of increasing metabolic acidosis and transient extreme bradycardia. The child made a prompt recovery. Treatment was continued with prostaglandin E, dipyridamole, and heparin.

Six days later the right femoral vein was catheterised and a $4 \mathrm{~F}$ Cobra catheter was passed to the right atrium, the right ventricle, across the ventricular septal defect to the ascending aorta, and thence to the arterial duct. A guide wire $(0.018$ inch Terumo) was positioned in the right lower lobe pulmonary artery and the catheter was exchanged for a $3.5 \mathrm{~mm}$ (3.6F) ACS Pinkerton balloon angioplasty catheter. During catheter manipulation through the right ventricle the stent became dislodged distally from the balloon, coming to lie on the guide wire alone high in the inferior caval vein. The stent was retrieved by snaring the distal end of the guide wire in the right atrium via a second catheter in the femoral vein. Retrieval of the stent was complicated by acute tamponade caused by cardiac perforation by the tip of the snare, but this was treated successfully by prompt pericardiocentesis and autotransfusion. The procedure was abandoned. The child made an uncomplicated recovery and eight days later the duct was again catheterised with a $6 \mathrm{~F}$ long curved sheath via the left femoral vein. The sheath was advanced to the ascending aorta and a guide wire was positioned in the right lower lobe pulmonary artery. A $15 \mathrm{~mm}$ stent was deployed in the duct with its tip just inside the existing $7 \mathrm{~mm}$ stent in the distal duct. Repeat angiography showed satisfactory stent positions with excellent flow to both pulmonary arteries, but the combined length of the stents still seemed to be too short. A further $7 \mathrm{~mm}$ stent was deployed in the most proximal part of the duct. Transient marked hypotension and bradycardia occurred at this stage, apparently owing to splinting of the right ventricle, the atrioventricular valve, and the aortic valve by the sheath. Final angiography showed a very satisfactory result.
Treatment was continued with dipyridamole, aspirin ( $15 \mathrm{mg} / \mathrm{kg} /$ day), and a heparin infusion to keep the activated partial thromboplastin time at 2-2.5 times the control value. The child remained well with arterial oxygen saturations above $80 \%$. He was extubated the following day and anticoagulated with warfarin, our initial plan being to continue this for eight weeks, when we expected that endothelialisation of the stent would be complete.

Ten days later he again became cyanosed. Repeat aortography via the right femoral artery showed very localised stenosis of the duct immediately at its junction with the pulmonary artery (fig 3). A further $7 \mathrm{~mm}$ stent was delivered to this site and dilated to a diameter of $3.5 \mathrm{~mm}$ without difficulty, and with prompt improvement in oxygen saturation to $90 \%$. At review eight days later the child was well and gaining weight. Pulse oximetry showed readings between $80 \%$ and $90 \%$ throughout the day. He was discharged home, where the following day he suffered two apnoeic episodes despite remaining pink and well. He suffered a further apnoeic episode on the way to hospital and resuscitation was unsuccessful. Necropsy showed that the duct was patent; there was asplenia and pneumococci were isolated from postmortem blood cultures. Three days later his twin brother was admitted to hospital after a near miss cot death at home.

\section{Discussion}

Although aortopulmonary shunt operations provide life-saving palliative treatment at low risk for neonates with pulmonary atresia, such surgical treatment has potential drawbacks. Both Blalock-Taussig (classical or modified) and Waterston shunts usually produce preferential blood flow to the pulmonary artery on the side of the shunt that can result in differential growth of the right and left pulmonary arteries. The Waterston anastomosis may be associated with distortion of the right pulmonary artery or pulmonary hypertension, and any kind of surgical intervention will inevitably result in scarring which may increase the risk of later, more definitive, surgical treatment. Non-surgical maintenance of ductal patency is therefore a highly desirable goal.

The recent development of stents for implantation into coronary arteries had led to the manufacture of stents of suitable size for use in the neonatal arterial duct. There are various forms of stent-from elaborate balloon expandable titanium coils to expandable stainless steel tubes and self expanding sprung steel cages. Self expanding stents have the potential disadvantage of relative lack of operator control of the final stent diameter. Balloon expandable stents have the theoretical advantage that it may prove possible to increase stent diameter at a later date simply by repeat balloon dilatation. We therefore thought that a balloon expandable stent was best suited to our purpose. Our initial choice of the Cook titanium coil design was prompted by the fact that the stent is clearly visible on radiographic screening. However, our experience suggests that a delivery system of $6 \mathrm{~F}$ is too large to allow safe 


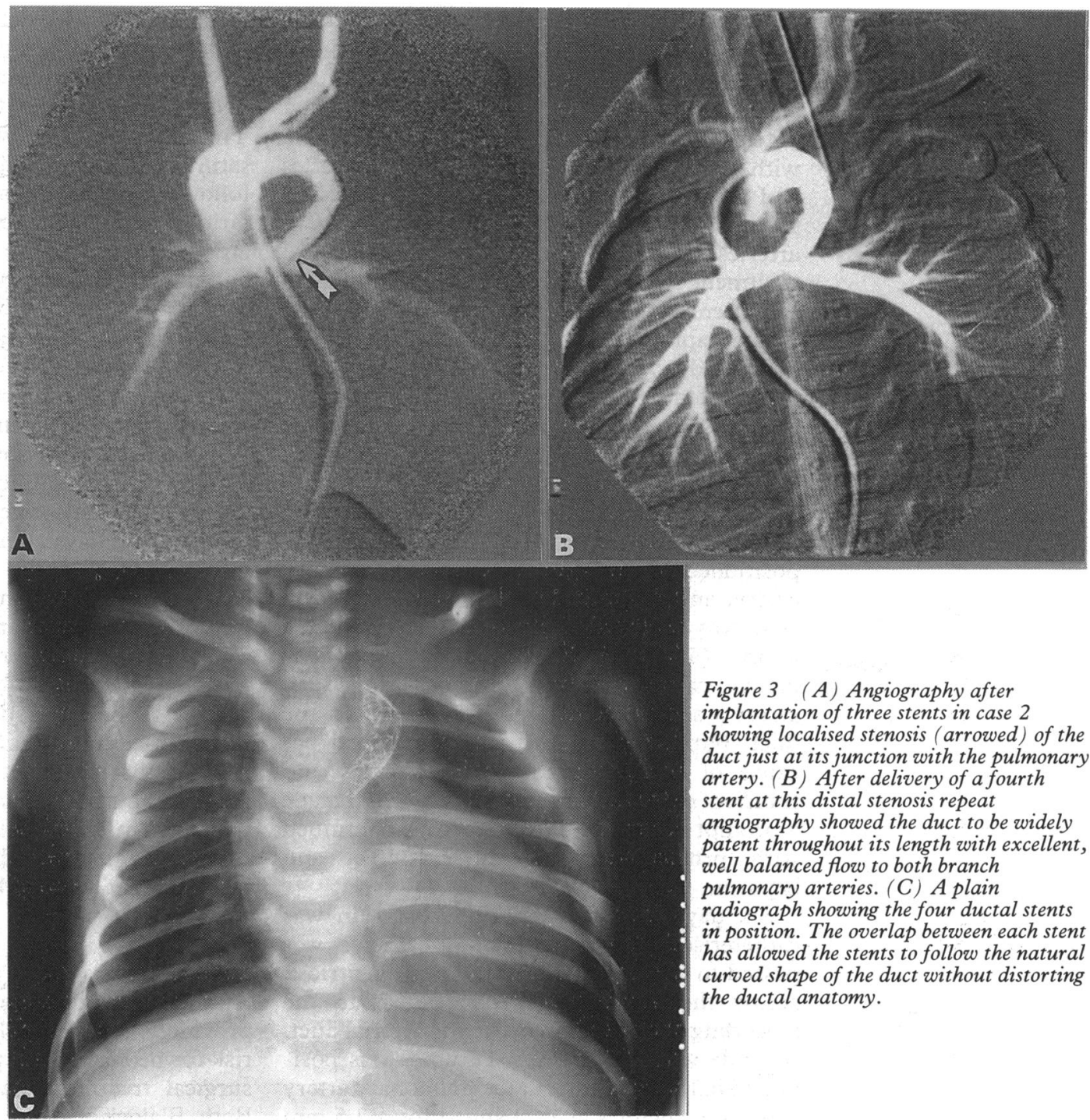

percutaneous arterial access in babies of this size. The Palmaz-Schatz stent has the distinct advantage of being operator mounted on the balloon catheter rather than being premounted by the manufacturer. This allows the operator to choose a balloon catheter of appropriate length, shaft size, and inflated diameter for individual ductal anatomy. The catheters we chose had shaft sizes of only $3 \cdot 3$, $3 \cdot 6$, and $3 \cdot 7 \mathrm{~F}$ and even with the stent mounted upon the balloon the catheter passed easily through a $5 \mathrm{~F}$ sheath which, in turn, was small enough to be introduced into the axillary artery with ease.

Despite the repeated cardiac catheterisations required in these cases before successful stenting of the duct, we encountered few major complications. Arterial damage should be avoidable if the stent delivery system can be kept as small as $5 \mathrm{~F}$. A peripheral right pulmonary artery was perforated by a superfloppy wire and the tip of a snare fashioned from a 0.014 inch guide wire perforated the heart. Heightened awareness of the possibility that even atraumatic wires can perforate the heart or blood vessels should minimise this risk.

It seems likely that the pulmonary haemorrhage in our first case was exacerbated by antiplatelet and anticoagulant therapy and in our case the bleeding required warfarin treatment to be stopped. Despite this there has been no suggestion of clot formation within the stent and it may be that the high flow through a $4 \mathrm{~mm}$ diameter duct makes thrombosis unlikely and anticoagulant therapy unneccessary. Treatment with aspirin and dipyridamole does, however, seem advisable in the light of experience with stent implantation at other sites, ${ }^{12}$ at least until the stent surfaces are likely to be completely endothelialised. We provisionally planned to continue these drugs for approximately two months from the stent implant. No complications were caused by ductal damage, although dissection of the duct by a guide wire or tears induced by stent deployment remain theoretical risks.

Repeated procedures were required in both our cases because short sections of the arterial duct remained unstented. This was partly because we decided to stent the ducts in sections, sometimes using half length stents to maximise flexibility of the balloon catheter/ stent combination at tortuous sites. The most distal part of the duct seems to have remarkable powers to constrict even when only a few millimetres is left unstented. We now appreciate that great care has to be taken to ensure very accurate stent positioning at this end of the 
duct at an initial procedure if repeated visits to the catheter laboratory are to be avoided.

There was no clear cause of death in our first case, but as far as is possible to ascertain neither child died with inadequate pulmonary blood flow. The first child had apnoeic attacks in the immediate neonatal period which had been attributed to airway obstruction related to his extensive cleft palate and it is possible that this may have contributed to his death. His pulmonary blood flow seemed to be relatively high and he had required treatment with diuretics; left ventricular volume overload may also have contributed to his death. The second child had extremely complex congenital heart disease and asplenia. His death was almost certainly due to septicaemia, because pneumococci were grown from the postmortem blood culture. His twin brother's near miss cot death is also of note but of uncertain relevance. We are planning further detailed investigation of the ducts, including histological assessment of endothelialisation.

Further follow up and further experience in other patients with a wider variety of ductal anatomy is clearly required before medium term results of ductal stenting can be assessed. At least in the short term, however, this procedure seems to promise a non-surgical alternative to aortopulmonary shunt surgery, with the very attractive benefit of providing balanced perfusion of both lungs and therefore the potential of balanced growth of the pulmonary arteries.

1 Mullins CE, O'Laughlin MP, Vick GW, et al. Implantation of balloon expandable intravascular grafts by catheterisation in pulmonary arteries and systemic veins. Circulation 1988;77:188-99.

2 Schatz RA, Baim DS, Leon $M$, et al. Clinical experience with the Palmaz-Schatz coronary stent. Initial results of a multicenter study. Circulation 1991;83:148-61. 